\title{
Construction of efficient xylose utilizing Pichia pastoris for industrial enzyme production
}

\author{
Pengfei $\mathrm{Li}^{1 \dagger}$, Hongbing Sun ${ }^{2 \dagger}$, Zao Chen ${ }^{1,3}$, Yin $\mathrm{Li}^{1}$ and Taicheng Zhu ${ }^{1 *}$
}

\begin{abstract}
Background: Cellulosic biomass especially agricultural/wood residues can be utilized as feedstock to cost-effectively produce fuels, chemicals and bulk industrial enzymes, which demands xylose utilization from microbial cell factories. While previous works have made significant progress in improving microbial conversion of xylose into fuels and chemicals, no study has reported the engineering of efficient xylose utilizing protein expression systems for the purpose of producing industrial enzymes.

Results: In this work, using Pichia pastoris as an example, we demonstrated the successful engineering of xylose metabolizing ability into of protein expression systems. A heterologous XI (xylose isomerase) pathway was introduced into P. pastoris GS115 by overexpressing the Orpinomyces spp. XI or/and the endogenous XK (xylulokinase) gene, and evolutionary engineering strategies were also applied. Results showed that the XI pathway could be functionally expressed in P. pastoris. After 50 generation of sequential batch cultivation, a set of domesticated recombinant P. pastoris strains with different performance metrics on xylose were obtained. One evolved strain showed the highest xylose assimilation ability, whose cell yield on xylose can even be comparable to that on glucose or glycerol. This strain also showed significantly increased $\beta$-mannanase production when cultured on xylose medium. Furthermore, transcription analysis of xylose pathway genes suggested that overexpression of XI and XK might be the key factors affecting effective xylose assimilation.
\end{abstract}

Conclusions: To our best knowledge, this study is the first work demonstrating the construction of efficient xylose utilizing P. pastoris strains, thus providing a basis for using cellulosic biomass for bulk industrial enzyme production.

Keywords: Xylose, Xylose isomerase, Recombinant Pichia pastoris, Evolutionary engineering, Industrial enzymes

\section{Background}

Growing energy crisis and environmental pressures led to renewed interest in cellulosic biomass as a renewable feedstock for the production of fuels and chemicals. In recent years, cellulosic biomass especially agricultural/ wood residues were exploited to produce value added products such as ethanol, xylitol, citric acids, lactic acid and other organic acids [1]. In addition to fuels and chemicals, there is also growing interest in utilization of agro-waste for fermentation of bulk enzymes including xylanase [2], lipase [3], cellulase [4], amylase [5] etc., which are needed in large volumes, but have a relatively

\footnotetext{
*Correspondence: zhutc@im.ac.cn

${ }^{\dagger}$ Equal contributors

${ }^{1}$ CAS Key Laboratory of Microbial Physiological and Metabolic Engineering, Institute of Microbiology, Chinese Academy of Sciences, Beijing 100101, China

Full list of author information is available at the end of the article
}

low unit value so that significantly lower manufacturing costs are demanded.

To date, one of the main problems impeding commercial conversion of cellulosic biomass into value added products is the inefficient microbial utilization of xylose [6,7], which is a major constituent of cellulosic biomass feedstock and the second most abundant carbohydrate in nature. This challenge can be addressed by engineering xylose metabolism in microbial cell factories with no or low xylose utilizing ability. Such work has been performed in a variety of industrial workhorse like Escherichia coli [8], Bacillus subtilis [9], Clostridium beijerinckii [10], Hansenula polymorpha [11], Corynebacterium glutamicum [12] with the aim of converting cellulosic biomass into biofuels and other useful chemicals. Most notably, in recent years, there have been an explosive of studies reporting construction of xylose utilizing Saccharomyces cerevisiae strains for cellulosic ethanol production [13-15]. Unfortunately, while previous 
works have made significant achievement in improving microbial conversion of xylose into chemicals, no study has reported the building of xylose metabolic pathway into protein expression system with the aim of cost-effectively producing industrial enzymes. Since protein production in enzyme producers is often growth-associated during protein expression phase $[16,17]$, producing strains which can most efficiently assimilate xylose into biomass are required. This is different from microbial conversion of xylose to chemicals, which is usually non-growth-associated and requires the maximum possible yield of product from the constructed strains.

In this work, as a proof-of-concept, we outlined the introduction of xylose utilization pathway into the enzyme producer Pichia pastoris. P. pastoris is one of the most successful eukaryotic expression systems developed in the past decade [18]. It showed great potential in the expression of a highly diverse of proteins and is most potent in the expression of several bulk enzymes, like phytase [19], mannanase [20], cellulase [21] and lipase [22]. Previous literature seems to indicate that $P$. pastoris are not able to utilize xylose as sole carbon source [23]. In this work, we induced a heterologous xylose pathway into $P$. pastoris by overexpressing the xylose isomerase (XI) from an anaerobic rumen fungus Orpinomyces spp. Combined with evolutionary engineering strategy, we finally were able to obtain a recombinant $P$. pastoris strain which can efficiently assimilate xylose for $\beta$-mannanase expression. This study is the first work demonstrating the construction of efficient xylose-utilizing $P$. pastoris strains.

\section{Results and discussion}

$P$. pastoris strain GS115 can assimilate xylose at slow rate Whether $P$. pastoris can utilize xylose as carbon source was rarely reported in previous literature. One study by Inan et al. concluded that $P$. pastoris are not able to utilize xylose as sole carbon source [23]. In this work, the ability of $P$. pastoris to assimilate xylose was investigated in the first place.

The $P$. pastoris strain GS115 was inoculated into complex media with and without the addition of xylose. Results showed that without the presence of carbon source, the complex medium (which was rich in oligopeptides) can only sustain the growth of yeast cells for less than $48 \mathrm{~h}$. Cell growth ceased at an $\mathrm{OD}_{600}$ of 14.5 and the cell mass began decreasing. In contrast, with the addition of xylose, cells kept growing for $120 \mathrm{~h}$ and reached a final $\mathrm{OD}_{600}$ of 28.1. Residue xylose concentration kept decreasing simultaneously and was depleted at $120 \mathrm{~h}$. These results indicated that $P$. pastoris strain GS115 was able to assimilate xylose as a carbon source (Figure 1). However, the growth rate of GS115 on xylose was very low with a specific growth rate of $0.0075 \mathrm{~h}^{-1}$ (which approximates to a doubling time of $92 \mathrm{~h}$; Table 1),

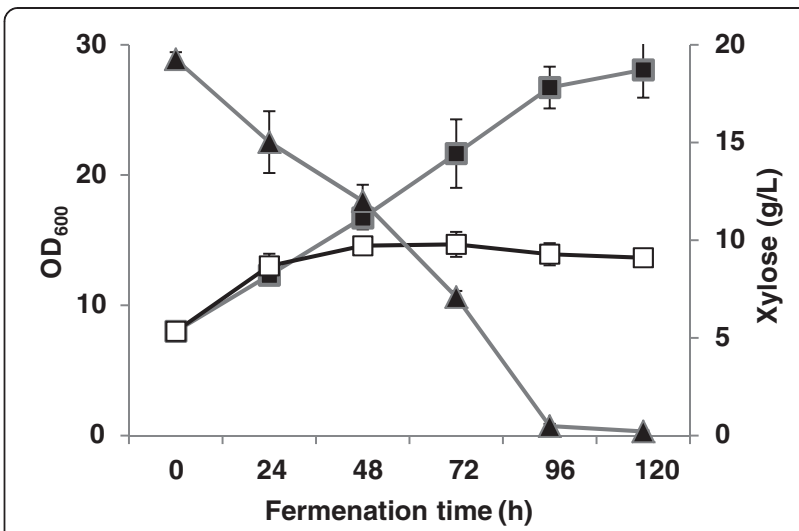

Figure 1 Fermentation profiles of Pichia pastoris strain GS115 cultured in complex media with and without xylose. Hollow square, cell growth without xylose; Solid square, cell growth with xylose; Solid triangle, residue xylose concentration. Three parallel flasks were tested.

which probably explains why xylose utilization has not been described for $P$. pastoris.

In yeast species, xylose metabolism was started by its conversion to xylulose, which was mediated by two consecutive enzymatic reactions catalyzed by NADPHdependent xylose reductase (XR) and $\mathrm{NAD}^{+}$-dependent xylitol dehydrogenase (XDH) (Figure 2). An examination over $P$. pastoris genome revealed an annotated gene (PAS_chr3_0744) encoding for putative XR and an unannotated gene (PAS_chr1-1_0490) homologous to XDH of other yeast species like Saccharomyces cerevisiae (56\% identity), Kluyveromyces marxianus (57\% identity), suggesting that $P$. pastoris genetically possesses the putative pathway for xylose assimilation. Further work such as genetic complementation tests are still required to validate this pathway.

\section{Introducing XI pathway alone did not significantly} enhance xylose assimilation ability of $P$. pastoris In order to enhance the xylose utilizing efficiency of $P$. pastoirs, we need to engineer an efficient xylose utilizing pathway in the host strain. In addition to the oxidoreductase (or XR/XDH) xylose pathway, another xylose pathway referred as XI pathway (which was found mainly in bacterial systems) can directly convert xylose to xylulose by xylose isomerase (XI) (Figure 2). The XI pathway is more desirable in xylose pathway engineering in recent years because it can eliminate the cofactor imbalance and the intermediate byproduct xylitol as normally observed in oxidoreductase xylose pathway. Therefore, in this work, we tried to enhance the flux from xylose towards pentose phosphate pathway (PPP) by introducing the XI pathway into $P$. pastoris.

The XI gene derived from Orpinomyces spp. was chosen because up to date only the XI from Piromyces and 
Table 1 Plasmids and strains used in present study

\begin{tabular}{|c|c|c|}
\hline Plasmids or strains & Relevant characteristics & Reference or source \\
\hline \multicolumn{3}{|l|}{ Plasmids } \\
\hline pGAPZmazF & Vector for constitutive secreted protein expression; Zeo ${ }^{r}$ & Invitrogen \\
\hline pPIC9K & Vector for inducible secreted protein expression; HIS4; kan $^{r}$ \& Ampr & Invitrogen \\
\hline pGAPZmazF (E,N) & pGAPZmazF based expression vector, containing EcoR I and Not I at multiple cloning site; Zeo ${ }^{r}$ & Our lab \\
\hline pGAPZH & pGAPZ based expression vector, carrying the HIS4 fragment from pPIC9K; Zeor & Our lab \\
\hline pGAPZ-XI-His & pGAPZ containing XI from Orpinomyces spp. and HIS4 fragment from pPIC9K; Zeor & This study \\
\hline PGAPZ-XK & pGAPZ containing XK from P. pastoris GS115; Zeo ${ }^{r}$ & This study \\
\hline pGAPKH-3Sman & pGAPKH containing three copies of alkaline $\beta$-mannanase gene, Kan ${ }^{r}$ & [33] \\
\hline \multicolumn{3}{|l|}{ Strains } \\
\hline E. coli DH5a & & Takara \\
\hline P. pastoris GS115 & his4 $4^{-}$, Mut $^{+}$ & Invitrogen \\
\hline Orpinomyces spp. & & Our lab \\
\hline GS-XI & GS115 integrated with the recombinant plasmid pGAPZ-XI-His & This study \\
\hline GS-XK & GS115 integrated with the recombinant plasmid pGAPZ-XK & This study \\
\hline GS-XI-XK & GS115 integrated with the recombinant plasmid pGAPZ-XI-His and pGAPZ-XK & This study \\
\hline GS115 & GS115 with 50 generations of evolution & This study \\
\hline $\mathrm{GS}-\left.\mathrm{XI}\right|^{\mathrm{SB} 50}$ & GS-XI with 50 generations of evolution & This study \\
\hline $\mathrm{GS}-\mathrm{XK}^{\mathrm{SB} 50}$ & GS-XK with 50 generations of evolution & This study \\
\hline GS-XI-XK SB50 & GS-XI-XK with 50 generations of evolution & This study \\
\hline GS-3Sman & GS115 integrated with the recombinant plasmid pGAPKH-3Sman & This study \\
\hline GS-XI-3Sman & GS-XI ${ }^{\mathrm{SB} 50}$ integrated with the recombinant plasmid pGAPKH-3Sman & This study \\
\hline
\end{tabular}

Orpinomyces [15] were functionally expressed in yeast system such as S. cerevisiae. In addition, previous work found that overexpression of xylulokinase (XK), which phosphorylates the xylulose to xylulose-5-phosphate (X5P) can also increase the flux towards the PPP [24]. Therefore, the Orpinomyces spp. XI gene (chemically synthesized, codon optimized) and endogenous putative $X K$ gene (encoded by PAS_chr1-1_0280, amplified by PCR) were both placed under the strong constitutive GAPDH promoter of $P$. pastoris, resulting in the recombinant vectors pGAPZ-XI-His

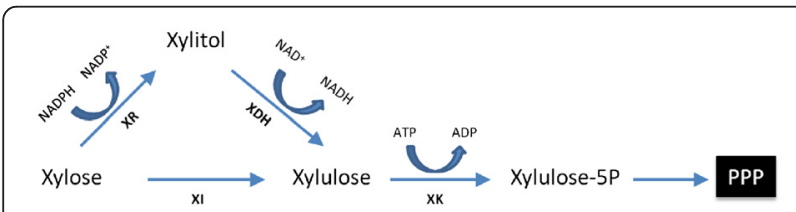

Figure 2 Xylose utilizing pathway in engineered Pichia pastoris. XR, putative xylose reductase (encoded by PAS_chr3_0744); XDH, putative xylitol dehydrogenase (encoded by PAS_chr1-1_0490); Xl, xylose isomerase (derived from Orpinomyces spp.); XK, putative xylulokinase (encoded by PAS_chr1-1_0280). PPP, pentose phosphate pathway. The $\mathrm{XR}$ and $\mathrm{XDH}$ consist of the putative oxidoreductase xylose pathway of $P$. pastoris. The XI xylose pathway is heterologously introduced by over-expression of Orpinomyces spp. XI gene in P. pastoris.
(Figure 3A) and pGAPZ-XK (Figure 3B). The two plasmids were separately or together transformed into GS115, thus generating three $P$. pastoris strains GS-XI, GS-XK and GS-XI-XK.

SDS-PAGE analysis showed that XI could be successfully overexpressed in $P$. pastoris, as shown in GS-XI and GS-XI-XK (Figure 3C), which was then confirmed by MALDI-TOF MS analysis. The expression of XK cannot be detected in any strains by SDS-PAGE analysis perhaps due to its low expression level while can be verified at transcriptional level by qPCR analysis (data not shown). The four strains GS115 (control), GS-XI, GSXK and GS-XI-XK were then tested for their xylose metabolizing ability using shake flask culture. Results showed that generally no significant difference in xylose fermentation profiles could be observed for GS-XI, GSXK and GS-XI-XK as compared with GS115 (Figure 4A, B). Only a slight increase in cell growth over GS115 could be seen for GS-XI and GS-XI-XK at approximately $72 \mathrm{~h}$ of fermentation (Figure 4A). These results suggested that introducing the XI pathway may not be enough to ensure efficient xylose assimilation in $P$. pastoris. Metabolic adaption of yeast cells to xylose metabolism may be necessary, which can readily achieved by laboratory evolution. 


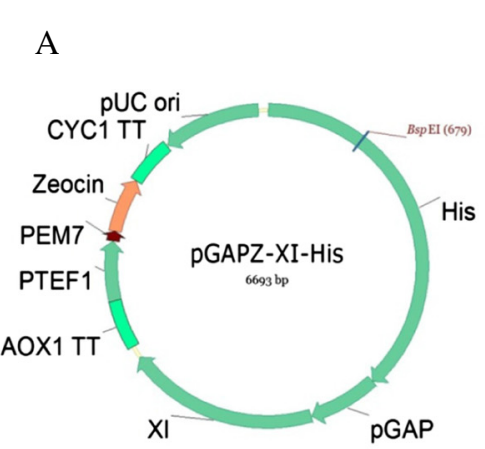

B

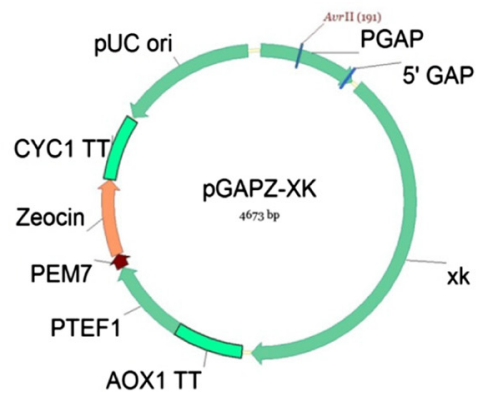

C

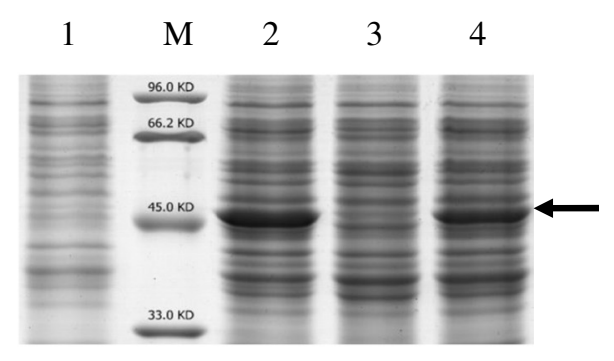

Figure 3 Expression of XI and XK in Pichia pastoris. (A) The expression vector containing the synthetic XI gene derived from Orpinomyces spp. (B) The expression vector containing the P. pastoris XK gene. (C) XI and XK expression analysis by SDS-PAGE. Lane 1, GS115 (control); Lane 2, GS-XI; Lane 3, GS-XK; Lane 4, GS-XI-XK; Lane M, protein molecular size marker. The right arrow indicate the position of XI.

\section{Xylose assimilation can be greatly improved by evolutionary engineering}

Evolutionary engineering strategy is widely applied to improve the pentose utilizing phenotype in yeast metabolic engineering $[13,25,26]$. The sequential batch cultivation method was used in this study with the aim of further improving xylose metabolizing ability of $P$. pastoris. After about 50 generations of evolution, we obtained evolved versions of GS115 ${ }^{\mathrm{SB} 50}, \mathrm{GS}-\mathrm{XI}^{\mathrm{SB} 50}, \mathrm{GS}-\mathrm{XK}^{\mathrm{SB} 50}$ and GS-XI-XK ${ }^{\mathrm{SB} 50}$, respectively. The domesticated strains were then evaluated for their abilities to metabolize xylose by comparing their fermentation profiles (Figure 5) and calculated performance metrics (Table 2), which included specific growth rate $(\mu)$, specific substrate consumption rate $\left(\mathrm{q}_{\mathrm{s}}\right)$ and cell yield on xylose $\left(\mathrm{Y}_{\mathrm{X} / \mathrm{S}}\right)$.

Results showed that evolutionary engineering remarkably improved the xylose utilizing performance of all the four recombinant strains as expected, but in opposite manners. On one hand, as shown for GS115 ${ }^{\mathrm{SB} 50}$, GS$\mathrm{XK}^{\mathrm{SB} 50}$ and GS-XI ${ }^{\mathrm{SB} 50}$, the cell growth were significantly improved on xylose medium after 50 generation of evolution (Figure 5A, B, C). The average specific growth rates of $\mathrm{GS}_{11} 5^{\mathrm{SB} 50}$, GS-XK ${ }^{\mathrm{SB} 50}$ and GS-XI ${ }^{\mathrm{SB} 50}$ were increased by $49 \%, 92 \%$ and $80 \%$, respectively (Table 2). GS-XI ${ }^{\mathrm{SB} 50}$ reached an $\mathrm{OD}_{600}$ of 45.2 after $96 \mathrm{~h}$ of fermentation, which represented the highest value of all studied strains (Figure 5B). Nevertheless, the specific xylose consumption rates of $\mathrm{GS} 115^{\mathrm{SB50}}, \mathrm{GS}-\mathrm{XK}^{\mathrm{SB} 50}$ and GS-XI ${ }^{\mathrm{SB} 50}$ were not improved or even slightly decreased (for GS-XI ${ }^{\mathrm{SB} 50}$ and $\mathrm{GS}-\mathrm{XK}^{\mathrm{SB} 50}$ ), which led to increased cell yield on xylose for the three strains. Especially for GS-XI ${ }^{\mathrm{SB50}}$, the $\mathrm{Y}_{\mathrm{X} / \mathrm{S}}$ on xylose reached a value of $0.378 \mathrm{~g} /$ g (Table 2), which can be comparable to that on glucose $(0.310 \mathrm{~g} / \mathrm{g})$ [27] or glycerol $(0.435 \sim 0.490 \mathrm{~g} / \mathrm{g})[27,28]$. The results suggested that a significant amount of xylose flux was assimilated into the biomass of GS-XI ${ }^{\mathrm{SB} 50}$, and construction of XI pathway combined with evolution engineering could be a powerful strategy to improve the xylose assimilation capability of $P$. pastoris.

On the other hand, as represented by GS-XI-XK ${ }^{\mathrm{SB} 50}$, the cell growth was not improved while the xylose utilization rate was significantly increased $\left(\mathrm{q}_{\mathrm{s}}\right.$ was increased by $56 \%$ ) after domestication (Figure 5D). As a result, the lowest $\mathrm{Y}_{\mathrm{X} / \mathrm{S}}$ on xylose $(0.120 \mathrm{~g} / \mathrm{g})$ was obtained for GS-XI-XK ${ }^{\mathrm{SB50}}$, which was only $32 \%$ of that of the GS$\mathrm{XI}^{\mathrm{SB} 50}$ (Table 2). The results suggested that the increase in xylose utilization for GS-XI-XK ${ }^{\mathrm{SB50}}$ was probably due to increased xylose dissimilation (such as respiration) in $P$. pastoris.

\section{Overexpression of XI and XK might be the key factors affecting the xylose assimilation}

Due to their essential role in xylose conversion, XI and XK would conceivably undergo significant changes 


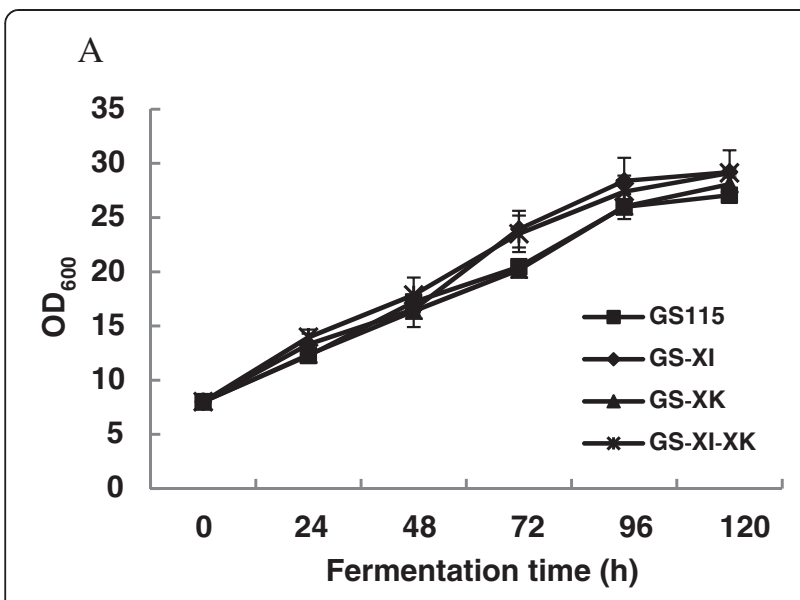

B

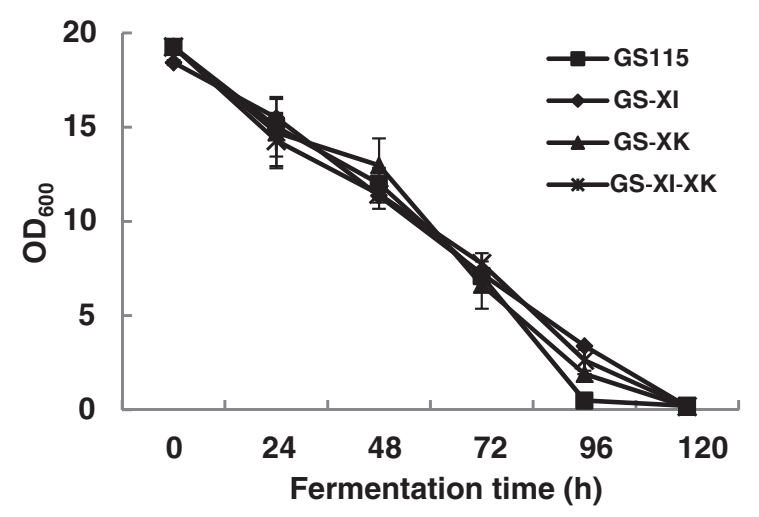

Figure 4 Fermentation profiles of three engineered $P$. pastoris strains on xylose with GS115 as a control. (A) Cell growth profile. (B) Xylose consumption profile. Three parallel flasks were tested for each stain.

during evolution process. Therefore, examining the changes in expression levels of $X I$ and $X K$ might provide useful information on how improvements in xylose metabolic characteristics occurred and thus help guide further rounds of strain engineering.

The $X I$ and $X K$ transcription levels in all four evolved strains together with their respective parental controls, were investigated by real time qPCR (Figure 6). Results showed $X I$ transcription was increased by $6 \sim 16$ fold in GS-XI $^{\mathrm{SB} 50}$ (Figure $6 \mathrm{~B}$ ) while $X K$ transcription was increased by $3.28 \sim 4.58$ fold in GS-XK ${ }^{\mathrm{SB} 50}$ (Figure $6 \mathrm{C}$ ). The remarkable up-regulation of $X I$ and $X K$ genes during evolution suggested that although already driven by the strong GAPDH promoter in the parent strains, the expression levels of XI and XK still could be the potential bottlenecks for effective xylose assimilation. This is especially true for XI, because despite the fact that XI had already achieved a considerably high expression level (accounting more than $4.5 \%$ of total soluble protein; Figure 3C) in the parent GS-XI strain (which would afflict yeast cells with great metabolic burden by occupying limited cellular resources), its expression level increased significantly after evolution, which illustrated the essential role XI plays in xylose assimilation. This conclusion was also in good consistence with a similar work performed in S. cerevisiae [26], where the authors by a comprehensive inverse metabolic engineering approach, concluded that elevated $X I$ expression level was responsible for the efficient xylose assimilation in evolved strain.

For GS-XI-XK ${ }^{\mathrm{SB} 50}$, the up-regulation of $X I$ or $X K$ as seen in GS-XK ${ }^{\mathrm{SB} 50}$ and GS-XK ${ }^{\mathrm{SB} 50}$ were not observed (Figure 6D), which was quite unexpected because GS$\mathrm{XI}-\mathrm{XK}{ }^{\mathrm{SB} 50}$ showed the highest xylose consumption rate among all strains. A possible explanation for this phenomenon was that the intrinsic oxidoreductase $\mathrm{xy}$ lose pathway of $P$. pastoris strain GS-XI-XK rather than the constructed XI pathway evolved during the sequential batch cultivation. This may also explain the observed low cell yield and increased dissimilation phenotypes for GS-XI-XK ${ }^{\mathrm{SB} 50}$ because The oxidoreductase xylose pathway can result in redox imbalance and thus interfere with normal cellular physiology [29]. In order to test this possibility, the transcription of $\mathrm{XR}$ and $\mathrm{XDH}$ were also determined using real-time qPCR. Results showed 4.9 11.1 fold increase in XDH mRNA levels in GS-XI$\mathrm{XK}^{\mathrm{SB} 50}$ (Figure 6D) while no significant difference in XR or XDH expression could be seen in other three evolved strains (data not shown). Therefore, increased XDH expression level could be responsible for increased xylose consumption rate in GS-XI-XK ${ }^{\mathrm{SB} 50}$, which was consistent with a previous study by Kim and his colleagues [30], where they observed that high level expression of $\mathrm{XDH}$ significantly increased xylose consumption rate (but not biomass yield) in S. cerevisiae.

In order to further investigate whether significant upregulation of xylose pathway genes in evolved strains was due to multi-copy integration events during evolution as described in evolved recombinant $S$. cerevisiae [26], the relative copy number changes of GS-XK ${ }^{\mathrm{SB50}}$ / GS-XK, GS-XI ${ }^{\mathrm{SB} 50} / \mathrm{GS}-\mathrm{XI}$ and GS-XI-XK ${ }^{\mathrm{SB} 50} / \mathrm{GS}-\mathrm{XI}-\mathrm{XK}$ were determined using qPCR. Results showed a six-fold change in XI copy number in GS-XI ${ }^{\mathrm{SB} 50}$ (Table 3). The enhancement of expression vector copy number in a single transformed cell line achieved by repeatedly plating it on high levels of a selectable marker drug, referred as post-transformational vector amplification (PTVA), has been previously described for P. pastoris [31], although the molecular mechanism is still unknown. This work demonstrated that in addition to drug marker system, PTVA also applied to other evolution pressure. In contrast, the up-regulation of XK in GS-XK and XDH in GS-XI-XK were not due to copy number increase (Table 3), which suggested more complex mechanisms might be involved in xylose pathway regulation. For a 


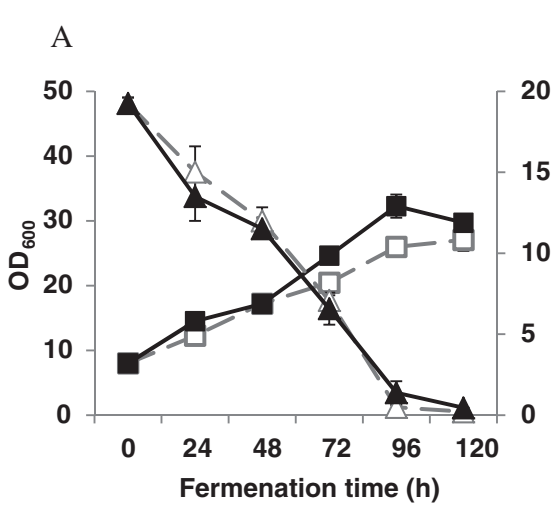

\section{B}

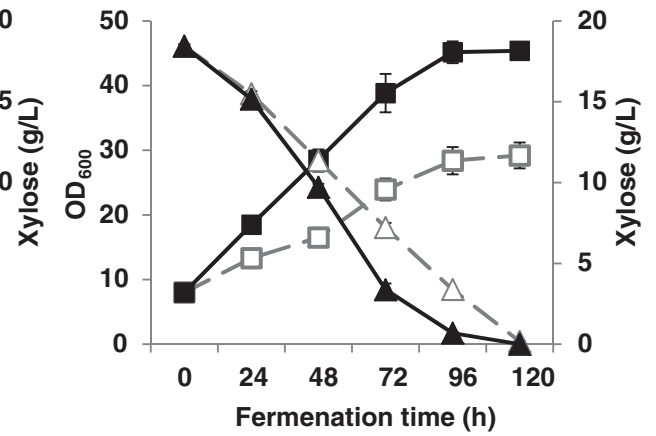

$\mathrm{C}$

$\mathrm{D}$
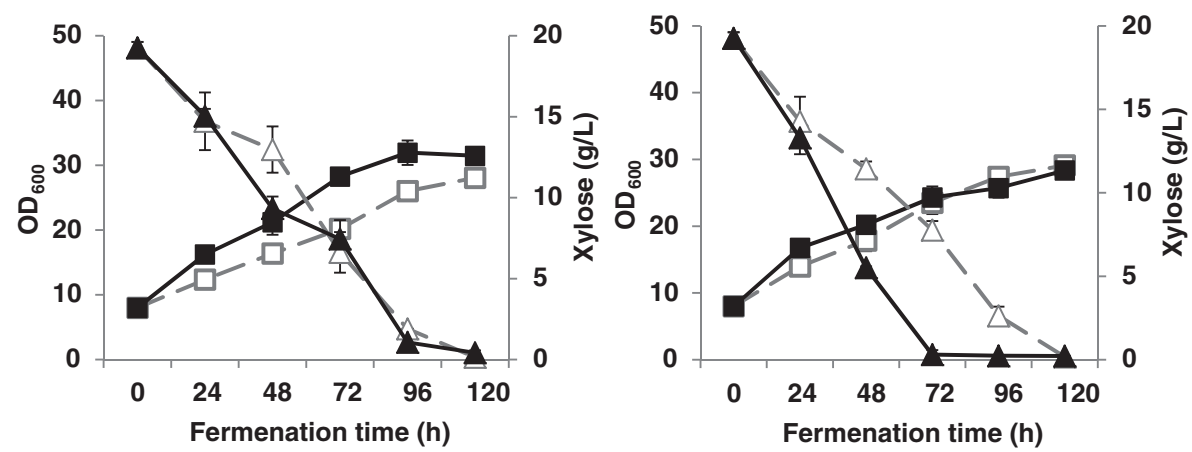

Figure 5 Fermentation profiles of engineered Pichia pastoris strains with 50 generations of evolution. (A) GS115 $5^{\mathrm{SB} 50}$. (B) GS-X ${ }^{\mathrm{SB} 50}$. (C) GS-XK ${ }^{\mathrm{SB} 50}$. (D) GS-XI-XK ${ }^{S B 50}$. The growth profiles of respective parental strains are also presented for comparison. Square, cell growth profile; Triangle, xylose consumption profile; Dashed line, the parental strains; Solid line, the evolved strains. All experiments were performed in triplicate.

comprehensive examination of genetic factors leading to the improved xylose assimilation phenotype, systems biotechnology tools such as comparative genomics, transcriptomics analysis need to be further applied.

Therefore, the overexpression of XI and XK may be important to obtain $P$. pastoris strains with high efficiency of

Table 2 The calculated fermentation performance metrics for all engineered Pichia pastoris strains

\begin{tabular}{llll}
\hline Strains & $\boldsymbol{\mu}\left(\mathbf{h}^{-\mathbf{1}}\right)$ & $\mathbf{q}_{\mathbf{s}}(\mathbf{g} / \mathbf{g ~ D C W} / \mathbf{h})$ & $\mathbf{Y}_{\mathbf{X} / \mathbf{s}}(\mathbf{g} / \mathbf{g ~ D C W})$ \\
\hline GS115 & $0.0075 \pm 0.0008$ & $0.056 \pm 0.006$ & $0.135 \pm 0.004$ \\
GS-XI & $0.0107 \pm 0.0014$ & $0.055 \pm 0.007$ & $0.194 \pm 0.007$ \\
GS-XK & $0.0072 \pm 0.0007$ & $0.059 \pm 0.008$ & $0.123 \pm 0.012$ \\
GS-XI-XK & $0.0103 \pm 0.0014$ & $0.057 \pm 0.008$ & $0.181 \pm 0.010$ \\
GS115 & $0.0112 \pm 0.0005$ & $0.061 \pm 0.006$ & $0.185 \pm 0.015$ \\
GS-XI & $0.0193 \pm 0.0013$ & $0.051 \pm 0.004$ & $0.378 \pm 0.013$ \\
GS-XK & $0.0138 \pm 0.0007$ & $0.051 \pm 0.006$ & $0.271 \pm 0.027$ \\
GS-XI-XK & $0.0110 \pm 0.0013$ & $0.092 \pm 0.011$ & $0.120 \pm 0.003$
\end{tabular}

$\mu$, specific growth rate; $q_{s}$, specific xylose consumption rate; $Y_{x / s}$, cell yield on xylose. All metrics were average values calculated based on fermentation data at $72 \mathrm{~h}$ of fermentation. The contribution of oligopeptides present in the complex medium to performance metrics was excluded based on data from Figure 1. xylose assimilation in further strain improvement. Since the XI has already reached an extremely high expression level, further work to find alternative XIs with higher catalytic efficiency such as proposed by Lee and his colleagues [32] was highly desirable.

\section{$\beta$-Mannanase expression was improved in xylose utilizing $P$. pastoris in xylose medium}

To investigate whether xylose utilizing phenotype would result in enhanced heterologous protein expression on xylose medium as our final aim, an alkaline $\beta$ mannanase [20] was taken as an example to evaluate the protein expression traits of GS-XI ${ }^{\mathrm{SB} 50}$. The expression plasmid pGAPKH-3Sman was transformed into GS-XI ${ }^{\text {SB50 }}$ and GS115 (used as a control), resulting in two recombinant strains GS-XI-3Sman and GS-3Sman, respectively. The two strains were first cultured with the BMGY medium in shaking-flasks. Results showed that two strains exhibited similar final cell biomass and $\beta$-mannanase production levels (Figure 7A), indicating that 50 generation of evolution did not affect the cell growth and enzyme expression profiles of engineered $P$. pastoris in glucose medium. When the two strains were 


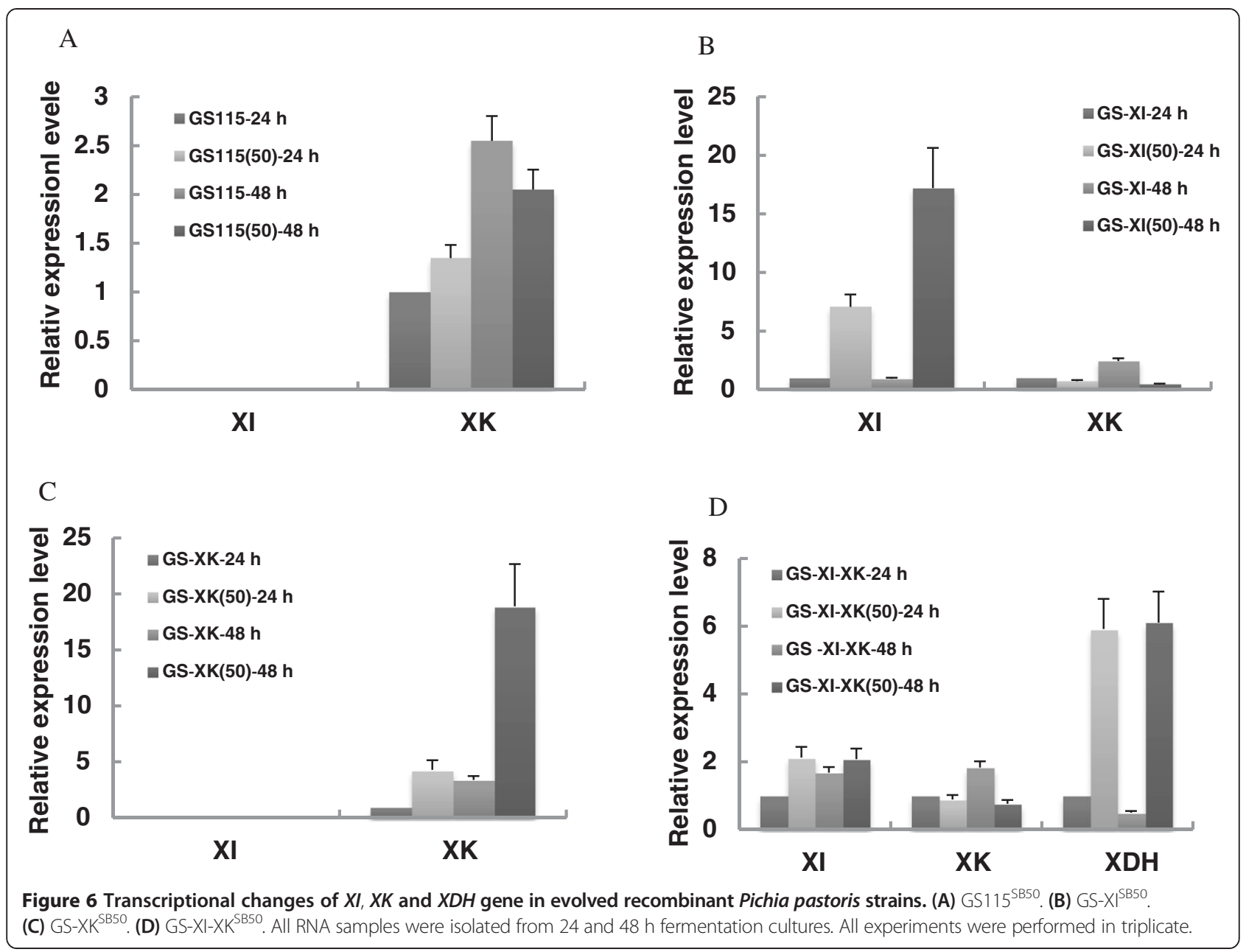

cultured with xylose medium (BMXY), the final biomass of GS-XI-3Sman is significantly higher than that of GS3 Sman (126\% increase) as expected. The final enzyme production of GS-XI-3Sman was $57.5 \%$ higher that of GS-3Sman (Figure 7B), showing that xylose utilizing $P$. pastoris is more productive in enzyme production (mainly due to increased cell growth) when cultured on xylose medium.

Table 3 Changes of copy numbers of xylose pathway genes after evolutionary engineering

\begin{tabular}{|c|c|c|c|}
\hline Strains & $\mathrm{XI}$ & $\mathrm{XK}$ & $\mathrm{XDH}$ \\
\hline$\overline{G S-X I} I^{S B 50} / G S-X I$ & $6.17 \pm 0.76$ & N.D & N.D \\
\hline GS-XK $K^{S B 50} / G S-X K$ & - & $0.84 \pm 0.27$ & N.D \\
\hline GS-XI-XK ${ }^{S B 50} / G S-X I-X K$ & $1.27 \pm 0.07$ & N.D & $1.03 \pm 0.19$ \\
\hline
\end{tabular}

Copy number was determined by real time qPCR. ACT1 gene was utilized as a reference control. Each sample was run in triplicates.

N.D: not determined, because no significant transcriptional changes were observed.

\section{Conclusions}

For the first time, we demonstrated the successful engineering of xylose metabolizing ability into $P$. pastoris for industrial enzyme production. A heterologous XI pathway was introduced into $P$. pastoris and evolutionary engineering strategy was also applied. A recombinant $P$. pastoris strain was finally obtained with the highest xylose assimilation ability, whose cell yield on xylose was nearly two-fold higher than that of the starting strain GS115. This strain also showed significantly increased $\beta$ mannanase production when cultured on xylose medium. This work provided a basis for construction of cell factories with the potential to cost-effectively produce bulk enzymes from cellulosic biomass.

\section{Methods}

\section{Strains and plasmids}

All the plasmids and the strains used in this work are listed in Table 1, and the primers are concluded in Table 4. Escherichia coli (E.coli) DH $5 \alpha$ and P. pastoris 


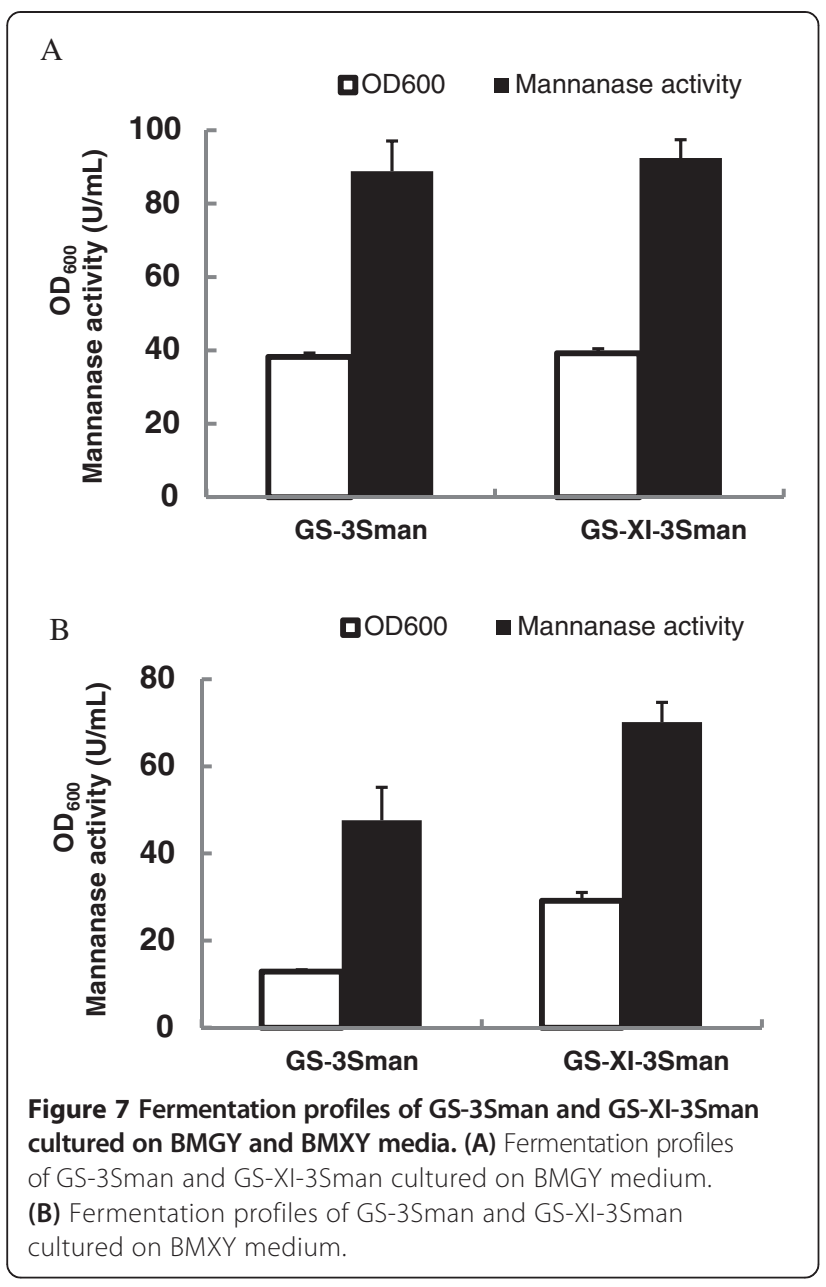

GS115 were routinely used for vector construction. All primers were synthesized by Invitrogen (Beijing, China).

\section{Growth and maintenance conditions}

E. coli strains were cultivated aerobically at $37^{\circ} \mathrm{C}$ in Luria-Bertani medium (10 g/L NaCl, $10 \mathrm{~g} / \mathrm{L}$ Tryptone (Thermo Fisher Oxoid, England) and $5 \mathrm{~g} / \mathrm{L}$ Yeast Extract (Thermo Fisher Oxoid, England), $\mathrm{pH} 7.4 \sim 7.6$ ) containing $25 \mathrm{mg} / \mathrm{L}$ Zeocin when required. All $P$. pastoris strains were grown at $30^{\circ} \mathrm{C}$ in YPD medium $(20 \mathrm{~g} / \mathrm{L}$ Glucose, $20 \mathrm{~g} / \mathrm{L}$ Peptone (Becton, Dickinson and Company, America) and $10 \mathrm{~g} / \mathrm{L}$ Yeast Extract) supplemented, when necessary, with $40 \mathrm{mg} / \mathrm{L}$ Zeocin for screening recombinant strains, while the HIS4 $4^{-}$recombinant $P$. pastoris strains were selected with MD solid medium (13.4 g/L YNB (Yeast Nitrogen Base w/o Amino Acid, Becton, Dickinson and Company, America), $0.4 \mathrm{mg} / \mathrm{L}$ Biotin, 20 g/L Glucose and 1.5\% Agar). All E. coli and P. pastoris strains were maintained frozen in $25 \%$ glycerol at $-80^{\circ} \mathrm{C}$. Complex culture media used in this work were BMXY (8.7 g/L monopotassium phosphate, $13.4 \mathrm{~g} / \mathrm{L} \mathrm{YNB}, 0.4$ mg/L Biotin, $20 \mathrm{~g} / \mathrm{L}$ Peptone, $10 \mathrm{~g} / \mathrm{L}$ Yeast Extract and
Table 4 Primers used in this study

\begin{tabular}{lll}
\hline Primer name & Primer sequences $\mathbf{( 5}^{\prime}$-3') & Size (bp) \\
\hline $5^{\prime}$ GAP & gtccctatttcaatcaattgaa & 22 \\
$3^{\prime}$ AOX & gcaaatggcattctgacatcc & 21 \\
XI-F & atcaagaattcatgactaagga & 22 \\
XI-R & aaagctggcggccgcttactgat & 23 \\
XK-F & atcggaattcatggttaccaaagaaatccaaa & 32 \\
XK-R & attggcggccgcaaacgcctgacttgcttcac & 32 \\
rACT1-F & agtgttcccatcggtcgtag & 20 \\
rACT1-R & ggtgtggtgccagatctttt & 20 \\
rXI-F & catacgttatgcgctgatgg & 20 \\
rXI-R & ccctcgctcactagatcgac & 20 \\
rXK-F & tcttcatggcaaggaggaac & 20 \\
rXK-R & atcgaagacggcatgatagg & 20 \\
rXR-F & taccatcaccctgacaacgt & 20 \\
rXR-R & atccaccaacctctctagcg & 20 \\
rXDH-F & cccgtctcgttacagcaatg & 20 \\
rXDH-R & gcatggacagcaacactcaa & 20 \\
\hline
\end{tabular}

Enzyme sites were in italics.

$20 \mathrm{~g} / \mathrm{L}$ Xylose, $\mathrm{pH}$ 6.0) and BMGY (identical to the BMXY except for the replacement of $20 \mathrm{~g} / \mathrm{L}$ Xylose with $20 \mathrm{~g} / \mathrm{L}$ glucose).

\section{Construction of recombinant plasmids}

The XI gene from Orpinomyces spp. was chemically synthesized in Genewiz, Inc. (Suzhou, China) with codon optimized. This fragment was treated with EcoRI and NotI and inserted into the intracellular expression vector pGAPZH, thus generating the recombinant plasmid pGAPZ-XI-His (Figure 3A). The endogenous $X K$ gene was cloned from genomic DNA of $P$. pastoris GS115 using XK-F and XK-R, creating EcoRI and NotI sites (italics in Table 4) and ligated to pGAPZmazF (E,N) to create the recombinant plasmids pGAPZ-XK (Figure 3B). The construction of the expression plasmid pGAPKH-3Sman, harboring three copies of alkaline $\beta$-mannanase gene was described in our previous work [33].

\section{Generation of recombinant $P$. pastoris strains}

The recombinant plasmid pGAPZ-XK was linearized by AvrII and transformed into P. pastoris GS115 and screened on the YPD agar plate with Zeocin according to the protocol of Invitrogen, and the resulting positive transformants verified by colony PCR were designated GS-XK. The recombinant plasmid pGAPZ-XI-His was linearized by BspEI and separately transformed into GS115 and GS-XK and screened on the MD agar plate, thus generating the recombinant $P$. pastoris strain GSXI and GS-XI-XK, respectively. 
The expression plasmid pGAPKH-3Sman was linearized by BspEI and transformed into GS-XI ${ }^{\mathrm{SB} 50}$ and GS115, resulting in two recombinant strains GS-XI3Sman and GS-3Sman, respectively.

\section{Shaking-flask fermentation}

Shaking-flask fermentation of the recombinant strains GS-XI, GS-XK and GS-XI-XK were performed with the GS115 as the control. All strains were first grown in YPD medium and then transferred into the BMXY medium with an initial $\mathrm{OD}_{600}$ of around 8. All cultures were performed at $30^{\circ} \mathrm{C}, 200 \mathrm{r} / \mathrm{m}$, for $120 \mathrm{~h}$. The cell concentration was determined from the $\mathrm{OD}_{600}$ value. Xylose concentration was analyzed by HPLC (HP1260; Agilent) using an Aminex HPX-87H ion-exchange column $(7.8 \times 300 \mathrm{~mm})$. The mobile phase was $0.05 \mathrm{mmol} /$ $\mathrm{L}$ sulfuric acid and the flow rate was $0.5 \mathrm{~mL} / \mathrm{min}$. The recombinant strain GS-XI-3Sman was cultured in the BMGY or BMXY at $30^{\circ} \mathrm{C}$ and $200 \mathrm{r} / \mathrm{m}$ for $96 \mathrm{~h}$, respectively, and the strain GS-3Sman used as a control.

\section{Evolutionary engineering of recombinant $P$. pastoris} Four strains GS115, GS-XI, GS-XK and GS-XI-XK were used to initiate the evolution process, which was performed by sequential batch cultivation under aerobic conditions in BMXY medium at $30^{\circ} \mathrm{C}$ with $200 \mathrm{r} / \mathrm{m}$. When the culture reached the stationary phase $(4 \sim 6 \mathrm{~d})$, an aliquot $(1 \mathrm{~mL})$ was transferred into a fresh $100 \mathrm{~mL}$ flask containing $25 \mathrm{~mL}$ BMXY liquid medium to start a new batch. The whole evolution process lasted for 50 generations (approximately one year), and the evolved strains were referred as GS-XI ${ }^{\mathrm{SB} 50}$, GS-XK ${ }^{\mathrm{SB} 50}$, GS-XI-XK ${ }^{\mathrm{SB} 50}$ and $\mathrm{GS} 115^{\mathrm{SB} 50}$, correspondingly.

\section{Genomic DNA preparation, RNA extraction and cDNA synthesis}

Genomic DNA was prepared by Bust n' Grab method [34]. Total RNA of each sample was prepared using an RNAprep pure kit (Tiangen Biotech, Co., Ltd, Beijing, China) following the manufacturer's recommendations. RNA samples were stored at $-80^{\circ} \mathrm{C}$ until used. $2 \mu \mathrm{g}$ of each total RNA was subjected to reverse transcription using the Fast Quant RT Kit (Tiangen Biotech, Co., Ltd, Beijing, China).

\section{Real-time qPCR for transcriptional analysis and copy number determination}

The PCR primer design was conducted using Primer3 software (http://primer3.ut.ee/). All the primers used in this work were listed in Table 4. The reaction conditions had been established as recommended by SYBR Premix ExTaq $^{\text {ma }}$ manual (TaKaRa Bio Co., Ltd, Dalian, China). Each $20 \mu \mathrm{L}$ reaction contained $10 \mu \mathrm{L} 2 \times$ SYBR Premix Ex $\mathrm{Taq}^{\mathrm{Tw}}, 0.4 \mu \mathrm{L} 50 \mu \mathrm{M}$ forward and reverse primers, $2.0 \mu \mathrm{L}$ sample cDNA, and 7.2 $\mu \mathrm{L}$ nuclease-free water. All realtime qPCR reactions were run in triplicate on a Light $\mathrm{Cy}-$ cler 96 (Roche, Switzerland) using the following program: $95^{\circ} \mathrm{C}$ for $3 \mathrm{~min}, 45$ cycles of $95^{\circ} \mathrm{C}$ for $5 \mathrm{~s}$, and $60^{\circ} \mathrm{C}$ for 20 s. The specificity of amplicons were verified by melting curve analysis after 40 cycles and agarose gel electrophoresis. The $2^{-\Delta \Delta}$ method was used for analyze $X I, X K, X R$ and $X D H$ gene in different strains with $A C T 1$ gene as the endogenous control (housekeeping gene). The copy numbers of genes $X I, X K$ and $X D H$ were determined following methods described previously [35].

\section{Protein analysis and enzyme activity determination}

Protein concentrations were determined using the Bradford method with bovine serum albumin (BSA) as a standard. Sodium dodecyl sulfate-polyacrylamide gel electrophoresis (SDS-PAGE) was performed on a $10 \%$ polyacrylamide gel with precision protein marker. To identify the target protein, protein bands in gel were excised and subjected to in-gel-digestion and matrix-assisted laser desorption ionization-time of flight mass spectrometry (MALDITOF MS) analysis. XI expression was estimated by optical density scanning using Gel-Pro Analyzer software (Media Cybemetics).

$\beta$-Mannanase activity was determined as described by $\mathrm{Ma}$ et al. [36]. One unit of $\beta$-mannanase activity was defined as the amount of enzyme that liberated $1 \mu \mathrm{mol}$ of reducing sugar per minute with locust bean gum as substrate.

\section{Competing interests}

The authors declare that they have no competing interests.

\section{Authors' contributions}

YL, TZ, LP and SH participated in the study design and in the writing of the manuscript. LP, SH and TZ performed the experiments. CZ participated in the construction of the expression plasmids. LP, SH, TZ and YL analyzed the data. All authors read and approved the final manuscript.

\section{Acknowledgements}

This research was supported by Beijing Municipal Natural Science Foundation (grant no. 5132024) and National Natural Science Foundation of China (no. 31000026).

\section{Author details}

${ }^{1}$ CAS Key Laboratory of Microbial Physiological and Metabolic Engineering, Institute of Microbiology, Chinese Academy of Sciences, Beijing 100101, China. ${ }^{2}$ National Engineering Laboratory for Industrial Enzymes, Tianjin Institute of Industrial Biotechnology, Chinese Academy of Sciences, Tianjin 300308, China. ${ }^{3}$ Department of Chemical and Biochemical Engineering, College of Chemistry and Chemical Engineering, Xiamen University, Xiamen 361005, China.

Received: 22 September 2014 Accepted: 6 February 2015 Published online: 21 February 2015

\section{References}

1. Chandel AK, Da Silva SS, Carvalho W, Singh OV. Sugarcane bagasse and leaves: foreseeable biomass of biofuel and bio-products. J Chem Technol Biot. 2012;87(1):11-20. 
2. Dos RL, Fontana RC, Delabona PS, Da SLD, Camassola M, Pradella JG, et al. Increased production of cellulases and xylanases by Penicillium echinulatum S1M29 in batch and fed-batch culture. Bioresour Technol. 2013;146:597-603.

3. Rodriguez JA, Mateos JC, Nungaray J, Gonzalez V, Bhagnagar T, Roussos S, et al. Improving lipase production by nutrient source modification using Rhizopus homothallicus cultured in solid state fermentation. Process Biochem. 2006:41(11):2264-9.

4. Bansal N, Tewari R, Soni R, Soni SK. Production of cellulases from Aspergillus niger NS-2 in solid state fermentation on agricultural and kitchen waste residues. Waste Manag. 2012;32(7):1341-6.

5. Rajagopalan G, Krishnan C. Hyper-production of alpha-amylase from agro-residual medium with high-glucose in SSF using catabolite derepressed Bacillus subtilis KCC103. J Basic Microbiol. 2010;50(4):336-43.

6. Jeffries TW. Engineering yeasts for xylose metabolism. Curr Opin Biotechnol. 2006;17(3):320-6

7. Van Vleet JH, Jeffries TW. Yeast metabolic engineering for hemicellulosic ethanol production. Curr Opin Biotechnol. 2009;20(3):300-6.

8. Chiang CJ, Lee HM, Guo HJ, Wang ZW, Lin LJ, Chao YP. Systematic approach to engineer Escherichia coli pathways for co-utilization of a glucose-xylose mixture. J Agric Food Chem. 2013;61(31):7583-90.

9. Chen T, Liu WX, Fu J, Zhang B, Tang YJ. Engineering Bacillus subtilis for acetoin production from glucose and xylose mixtures. J Biotechnol. 2013;168(4):499-505.

10. Xiao H, Li Z, Jiang Y, Yang Y, Jiang W, Gu Y, et al. Metabolic engineering of D-xylose pathway in Clostridium beijerinckii to optimize solvent production from xylose mother liquid. Metab Eng. 2012;14(5):569-78.

11. Dmytruk OV, Voronovsky AY, Abbas CA, Dmytruk KV, Ishchuk OP, Sibirny AA. Overexpression of bacterial xylose isomerase and yeast host xylulokinase improves xylose alcoholic fermentation in the thermotolerant yeast Hansenula polymorpha. FEMS Yeast Res. 2008;8(1):165-73.

12. Buschke N, Schroder H, Wittmann C. Metabolic engineering of Corynebacterium glutamicum for production of 1,5-diaminopentane from hemicellulose. Biotechnol J. 2011;6(3):306-17.

13. Cai Z, Zhang B, Li Y. Engineering Saccharomyces cerevisiae for efficient anaerobic xylose fermentation: reflections and perspectives. Biotechnol J. 2012;7(1):34-46.

14. Hahn-Hagerdal B, Karhumaa K, Jeppsson M, Gorwa-Grauslund MF. Metabolic engineering for pentose utilization in Saccharomyces cerevisiae. Adv Biochem Eng Biotechnol. 2007;108:147-77.

15. Matsushika A, Inoue $H$, Kodaki T, Sawayama S. Ethanol production from xylose in engineered Saccharomyces cerevisiae strains: current state and perspectives. Appl Microbiol Biotechnol. 2009;84(1):37-53.

16. Gheshlaghi R, Scharer JM, Moo-Young M, Douglas PL. Metabolic flux analysis for optimizing the specific growth rate of recombinant Aspergillus niger. Bioprocess Biosyst Eng. 2007;30(6):397-418.

17. Jungo C, RE Rat C, Marison IW, von Stockar U. Quantitative characterization of the regulation of the synthesis of alcohol oxidase and of the expression of recombinant avidin in a Pichia pastoris Mut ${ }^{+}$strain. Enzyme Microb Tech. 2006:39(4):936-44.

18. Damasceno LM, Huang CJ, Batt CA. Protein secretion in Pichia pastoris and advances in protein production. Appl Microbiol Biotechnol. 2012;93(1):31-9.

19. Bai Y, Yang P, Wang Y, Shi P, Luo H, Meng K, et al. Phytase production by fermentation of recombinant Pichia pastoris in monosodium glutamate wastewater. World J Microb Biot. 2009;25(9):1643-9.

20. Zhu T, You L, Gong F, Xie M, Xue Y, Li Y, et al. Combinatorial strategy of sorbitol feeding and low-temperature induction leads to high-level production of alkaline beta-mannanase in Pichia pastoris. Enzyme Microb Technol. 2011;49(4):407-12.

21. Mellitzer A, Weis R, Glieder A, Flicker K. Expression of lignocellulolytic enzymes in Pichia pastoris. Microb Cell Fact. 2012;11:61.

22. Eom GT, Lee SH, Song BK, Chung KW, Kim YW, Song JK. High-level extracellular production and characterization of Candida antarctica lipase B in Pichia pastoris. J Biosci Bioeng. 2013;116(2):165-70.

23. Inan M, Meagher MM. Non-repressing carbon sources for alcohol oxidase (AOX1) promoter of Pichia pastoris. J Biosci Bioeng. 2001;92(6):585-9.

24. Jeffries TW, Jin YS. Metabolic engineering for improved fermentation of pentoses by yeasts. Appl Microbiol Biotechnol. 2004;63(5):495-509.

25. Peng B, Shen $Y$, Li X, Chen $X$, Hou J, Bao X. Improvement of xylose fermentation in respiratory-deficient xylose-fermenting Saccharomyces cerevisiae. Metab Eng. 2012;14(1):9-18.
26. Zhou H, Cheng JS, Wang BL, Fink GR, Stephanopoulos G. Xylose isomerase overexpression along with engineering of the pentose phosphate pathway and evolutionary engineering enable rapid xylose utilization and ethanol production by Saccharomyces cerevisiae. Metab Eng. 2012;14(6):611-22.

27. Garcia-Ortega X, Ferrer P, Luis Montesinos J, Valero F. Fed-batch operational strategies for recombinant Fab production with Pichia pastoris using the constitutive GAP promoter. Biochem Eng J. 2013;79:172-81.

28. Wang Y, Wang Z, Du G, Hua Z, Liu L, Li J, et al. Enhancement of alkaline polygalacturonate lyase production in recombinant Pichia pastoris according to the ratio of methanol to cell concentration. Bioresour Technol. 2009:100(3):1343-9.

29. van Maris AJ, Winkler AA, Kuyper M, de Laat WT, van Dijken JP, Pronk JT. Development of efficient xylose fermentation in Saccharomyces cerevisiae: xylose isomerase as a key component. Adv Biochem Eng Biotechnol. 2007;108:179-204.

30. Kim SR, Ha SJ, Kong II, Jin YS. High expression of XYL2 coding for xylitol dehydrogenase is necessary for efficient xylose fermentation by engineered Saccharomyces cerevisiae. Metab Eng. 2012;14(4):336-43.

31. Sunga AJ, Tolstorukov I, Cregg JM. Posttransformational vector amplification in the yeast Pichia pastoris. FEMS Yeast Res. 2008;8(6):870-6.

32. Lee SM, Jellison T, Alper HS. Directed evolution of xylose isomerase for improved xylose catabolism and fermentation in the yeast Saccharomyces cerevisiae. Appl Environ Microbiol. 2012;78(16):5708-16.

33. Zhu T, Sun H, Li P, Xue Y, Li Y, Ma Y. Constitutive expression of alkaline $\beta$-mannanase in recombinant Pichia pastoris. Process Biochem. 2014;49 (12):2025-9.

34. Harju S, Fedosyuk H, Peterson KR. Rapid isolation of yeast genomic DNA: Bust n' Grab. BMC Biotechnol. 2004:4:8.

35. Zhu T, Guo M, Tang Z, Zhang M, Zhuang Y, Chu J, et al. Efficient generation of multi-copy strains for optimizing secretory expression of porcine insulin precursor in yeast Pichia pastoris. J Appl Microbiol. 2009;107(3):954-63.

36. Ma Y, Xue Y, Dou Y, Xu Z, Tao W, Zhou P. Characterization and gene cloning of a novel beta-mannanase from alkaliphilic Bacillus sp. N16-5. Extremophiles. 2004;8(6):447-54.

\section{Submit your next manuscript to BioMed Central and take full advantage of:}

- Convenient online submission

- Thorough peer review

- No space constraints or color figure charges

- Immediate publication on acceptance

- Inclusion in PubMed, CAS, Scopus and Google Scholar

- Research which is freely available for redistribution 